\title{
Evaluation of Effect of $0.2 \%$ Chlorhexidine, $0.2 \%$ Magnetized Chlorhexidine and Magnetized RO Water Mouth Rinse on Streptococcus Mutans in Children: A Prospective Cross-Sectional Study
}

Deepak Bhayya' Rajeev Prajapati ${ }^{1}$ Tarulatha R Shyagali² Prabhath Singh ${ }^{1}$ Saurabh Tiwari ${ }^{1}$

\author{
Address for correspondence: Tarulatha R Shyagali. MDS, DCE, \\ DNHE, PhD, Department of Orthodontics and Dentofacial \\ Orthopedics, Hitkarini Dental College and Hospital, Jabalpur, \\ Madhya Pradesh 482005, India \\ E-mail: drtarulatha@gmail.com
}

\begin{abstract}
Objective: To compare efficacy of commercially available $0.2 \%$ chlorhexidine mouth rinse, conventionally prepared magnetized water and $0.2 \%$ magnetized chlorhexidine on reduction of $S$. mutans colony count.

Materials and Methods: A cross-sectional prospective study was performed on a group of 30 children of age 11-12 years. The study was conducted over a period of 1 week. Sample was equally distributed amongst the 3 groups $(n=10)$ depending upon type of mouth rinse usage viz; Group I: $0.2 \%$ chlorhexidine mouthwash, Group II: 72 hours magnetized RO water and Group III: 72 hours magnetized $0.2 \%$ chlorhexidine mouthwash. Each group was asked to use $10 \mathrm{~mL}$ of the mouth rinse for 3 minutes once in a day for a period of 7 days. Saliva samples were collected at baseline, $3^{\text {rd }}$ day and $7^{\text {th }}$ day. Microbiological analysis of saliva samples was done for streptococcus mutant count on the inoculated cultural plates. One-way ANOVA test was applied to know the difference between the groups, further Tukey's test was performed for multiple comparison.

Results: There was a significant reduction in $S$. mutans count in plaque after 7 days from baseline in all the three groups. Mean colony forming units at day 7 was $181.60,185.80,149.80$ for $0.2 \%$ chlorhexidine mouthwash, magnetized water and magnetized $0.2 \%$ chlorhexidine mouthwash group respectively. The difference seen is statistically significant with magnetized $0.2 \%$ chlorhexidine mouthwash showing increased reduction in bacterial colony count.

Conclusion: Magnetically activated $0.2 \%$ chlorhexidine mouth wash is more potent in reducing the colony count in comparison to $0.2 \%$ chlorhexidine and magnetized water. Magnetized water can also be used as an environmental friendly mouth wash alternative to $0.2 \%$ chlorhexidine because of its comparable antibacterial efficacy.
\end{abstract}

Keywords: Antimicrobial efficacy, magnetized water, magnetized chlorhexidine, saliva, Streptococcus mutans

How to cite this article: Bhayya B, Prajapati R, Shyagali TR, Singh P, Tiwari S. Evaluation of Effect of $0.2 \%$ Chlorhexidine, $0.2 \%$ Magnetized Chlorhexidine and Magnetized RO Water Mouth Rinse on Streptococcus Mutans in Children: A Prospective Cross-Sectional Study. J Pediatr Dent 2021;7(2):65-71

OPEN ACCESS This work is licensed under a Creative Commons Attribution-NonCommercial 4.0 International License. 


\section{Introduction}

The dysbiosis in caries microbiome plays a critical role and is the primary etiology for dental caries. Understanding the dysbiosis of the biofilm focus on selected acidogenic and aciduric caries-associated species and helps in favorable modulation of the biofilm as a whole.[1]

Common mode of removing cariogenic pathogens by mechanical means like toothbrushes have been found to be insufficient. Thus, in addition to mechanical modes certain chemical control measures are opted. The mouthwashes are one of them. [2,3]

Despite the proven antimicrobial activity of chlorhexidine, number of local side effects such as, staining on the tooth restorative materials, loss of taste sensation, oral mucosal erosion, calculus deposition, parotid duct stenosis, hypersensitivity, and bitter taste makes it a non-ideal mouth wash.[4] Further, chlorhexidine mouthwashes cannot have long-term effects on oral bacteria[5] and $S$. mutans increases again after weeks or months.[2] This finding shows the need for the development of a new antibacterial agent that can work against the microbiological burden of streptococcus mutant and which can also be oral tissue friendly, so that children can use it without having to compromise their gingival health.[6]

Some of the new natural antibacterial mouthwash like, adding salt to warm water helps in gum irritations and thus helps in healing after tooth or gum procedures. Few drops of different essential oils can target bad breath and bacteria that lead to gingivitis and tooth decay. Magnetized water raises the $\mathrm{pH}$ level of saliva and gets rid of the toxins. It is an energy-building, activating, cleansing, bacteriostatic and bactericidal agent.[7]

Magnets in health field are used for various purposes and they are known for their efficiency to prevent the disease from occurring. Magnets when come in contact with the water, are capable of changing the properties of the hydrogen ions and the other minerals present in water, thus making the water to become magnetized. This magnetized water can be beneficial for the human health. Literature says that for reaping the benefits of the magnetized water, one has to use it immediately after the preparation. $[8,9]$

Ferrite magnet is known to inhibit the growth of many variants of bacteria and its effect are strength dependent.[10] The potential uses of magnets in the field of dentistry are not been explored completely. Keeping this in mind, the current study was designed to understand the effect of magnetized water and magnetized chlorhexidine in reducing microbial load.

\section{Materials and Methods}

A prospective cross sectional study was performed on a group of 30 children aged between 11-12 years. Ethical clearance was obtained by the institutional ethical committee. Power sampling was done before deciding the final sample based on the population size of the previous studies of similar nature. Before the start of the study, official permission was obtained from the District Educational Officer and the school headmaster of the children who were given an informational document describing the study proposal and the role performed by each participant. The purpose of the study was also explained to the parents of the children and the singed inform consent was taken from them for their willing participation in this study. The study was conducted over a period of a week. The sample selection was done using the following preset selection criteria:

\section{Inclusion criteria}

- Subjects who were in mixed dentition period,

- DMFT score $>4$

- Systemically healthy patients

- No orthodontic appliances or removable prosthesis

- No history of antibiotic therapy, oral rinses or oral prophylaxis within previous 3 months.

\section{Exclusion criteria}

- History of fluoride treatment in the past 2 weeks

- Eating 1 to 2 hours before sample collection

- Physically and mentally retarded Children

- Children who exhibited pathologies related to oral cavity

After the fulfillment of the selection criteria, 30 children were selected who were divided into 3 equal groups,

Group I $(n=10)$ : Control $0.2 \%$ Chlorhexidine mouthwash (Thermokind mouth wash, Mankind Pharma, India).

Group II $(n=10): 72$ hours magnetized Reverse Osmosis (RO) water

Group III $(n=10): 72$ hours magnetized $0.2 \%$ Chlorhexidine mouthwash (Thermokind mouth wash, Mankind Pharma, India).

Initially, all children underwent the scaling and polishing of the teeth using the ultrasonic scaler. Followed by which, they were instructed not use any oral hygiene practices for 24 hours. After which, baseline saliva sample was collected. 
Table 1. Properties of the mouth washes

\begin{tabular}{|l|c|c|c|c|}
\hline Type of property & RO water & $\mathbf{0 . 2 \%}$ chlorhexidine & $\mathbf{7 2}$ hrs. magnetized & 72 hrs. magnetized \\
\hline & (normal) & mouthwash, & RO water, & $\mathbf{0 . 2 \%}$ chlorhexidine \\
\hline $\mathrm{pH}$ & & & & mouthwash \\
\hline Electrical conductivity & 7.20 & 4.24 & 7.98 & 5.63 \\
\hline
\end{tabular}

\section{Saliva sample collection}

Saliva was selected using the spitting method in a sterile sample collecting plastic vials for all 3 groups. Culture vials were taken out at room temperature 1 hour before the sample collection. The unstimulated saliva sample was collected in the morning between 10 -12 am matching the circadian rhythm, to check for the $S$. mutans count. Care was also taken to avoid saliva collection before heavy physical exercise. Then were carried in the ice box containing ice to the laboratory (Excellent Bio Research Solutions Pvt. Ltd.), there the inoculation was performed for the streptococcus mutant count on the cultural plate. Subsequently, after the collection of the baseline salivary sample, the children were given instruction group wise to use the respective prescribed mouth rinses under the supervision of their parents or the guardians. Later the salivary sample were collected on day 3 and day 7.

Sample collection schedule;

Day 1 - Baseline morning 10 - 12 am

Day 3 - Morning 10 - 12 am

Day 7 - Morning 10 - 12 am

\section{Magnetization of $\mathrm{RO}$ water and $0.2 \%$ chlorhexidine mouth rinse}

In two vials of $100 \mathrm{~m} \mathrm{~L}$ glass beakers, reverse osmosis water and $0.2 \%$ chlorhexidine mouth rinse was taken. Both the glass beakers were kept over the magnets for the duration of 72 hours, so that both the sample could get magnetized. All the three mouth rinses viz, $0.2 \%$ chlorhexidine mouth rinse, magnetized $\mathrm{RO}$ water and magnetized $0.2 \%$ chlorhexidine mouth rinse, were sent to a local chemical laboratory (Excellent Bio Research Solutions Pvt. Ltd.) to verify their magnetization. In the laboratory, mouth rinses were checked for their electrical conductivity and $\mathrm{PH}$, the report of the same is depicted in Table 1. The culture media used was Mutans-sanguis agar (Himedia). The sample was homogenized by centrifugation. Serial dilution of sample was made with sterile saline. Hundred micro liters of diluted sample was spread on Mitis-salivariusbacitracin agar (Himedia) by a glass spreader. Plates were incubated at $37^{\circ} \mathrm{C}$ for 48 hours in anaerobic jar. Bacterial colonies were observed using a dissecting microscope and deduced on the basis of colony morphology, shape, and color. Smears were performed from colonies and stained with gram stain. Colony counting was done under a colony counter and number of colony forming units (CFU) were multiplied by dilution factor and expressed as number of $\mathrm{CFU} / \mathrm{ml}$ of sample.

Collected data was tabulated and was amended to statistical analysis using SPSS version 22 software. To check the normality of data Kolmogorov-Smirnov and Shapiro-Wilk test were used, the results of which showed $\mathrm{p}>0.05$ (non-significant) depicting that the data is normal. So, it was decided to use the parametric test; One-way ANOVA test was used to check the difference for the colony count in different groups, and Tukey's test was used for the multiple comparison.

\section{Results}

Table 2 depicts the data pertaining to the difference in the colony forming units for different groups at different time periods. At day 0, mean values for CFU count was maximum in magnetized water i.e. $233.80 \pm 33.34$ followed by Magnetized $0.2 \%$ chlorhexidine $(233.80 \pm 27.17)$ and $0.2 \%$ chlorhexidine $(228.60 \pm 27.51)$. This difference in the mean values were found to be non-significant ( $\mathrm{p}$ value $=0.914$ ). At day 3 , maximum mean values for CFU count was in magnetized water, followed by $0.2 \%$ chlorhexidine and minimum in magnetized $0.2 \%$ chlorhexidine with non-significant difference $(p=0.746)$. Similarly, the mean values at day 7 was maximum in magnetized water and minimum in magnetized $0.2 \%$ chlorhexidine. The difference noted was significant $(\mathrm{p}=0.005)$.

Table 3 represents intragroup comparison at each interval. Day 0 and day 3 showed insignificant values for all the groups. But significant difference was noted at day 7 with the mean difference of -36.00 between magnetized $0.2 \%$ chlorhexidine and magnetized water and mean difference of 4.2 between magnetized water and $0.2 \%$ chlorhexidine. 
Table 2. Intergroup comparison between different groups on the basis of CFU count at day 0, 3 and 7

\begin{tabular}{|c|c|c|c|c|c|}
\hline Groups & Mean & SD & Mean square & F value & $\mathbf{p}$ \\
\hline \multicolumn{6}{|l|}{ Day 0} \\
\hline $0.2 \%$ chlorhexidine & 228.60 & 27.51 & 78.40 & 0.09 & 0.914 \\
\hline Magnetized water & 233.80 & 33.34 & & & \\
\hline Magnetized $0.2 \%$ chlorhexidine & 233.00 & 27.17 & & & \\
\hline \multicolumn{6}{|l|}{ Day 3} \\
\hline $0.2 \%$ chlorhexidine & 202.60 & 23.24 & 122.80 & 0.29 & 0.746 \\
\hline Magnetized water & 206.40 & 22.76 & & & \\
\hline Magnetized $0.2 \%$ chlorhexidine & 199.40 & 13.56 & & & \\
\hline \multicolumn{6}{|l|}{ Day 7} \\
\hline $0.2 \%$ chlorhexidine & 181.60 & 22.67 & 3874.80 & 6.54 & $0.005^{*}$ \\
\hline Magnetized water & 185.80 & 22.54 & & & \\
\hline Magnetized $0.2 \%$ chlorhexidine & 149.80 & 27.47 & & & \\
\hline
\end{tabular}

Test applied: One-way ANOVA; $\mathrm{p} \leq 0.05$ (Significant), SD: Standard deviation

Table 3. Intragroup comparison between different groups on the basis of CFU count at day 0, 3 and 7

\begin{tabular}{|c|c|c|c|c|c|c|}
\hline \multirow[t]{2}{*}{ (I) Groups } & \multirow[t]{2}{*}{ (J) Groups } & \multirow{2}{*}{$\begin{array}{c}\text { Mean difference } \\
\text { (I-J) }\end{array}$} & \multirow[t]{2}{*}{ Std. error } & \multirow[t]{2}{*}{ Sig. } & \multicolumn{2}{|c|}{ 95\% Confidence Interval } \\
\hline & & & & & Lower bound & Upper bound \\
\hline \multicolumn{7}{|l|}{ Day 0} \\
\hline \multirow[t]{2}{*}{$0.2 \%$ chlorhexidine } & Magnetized $0.2 \%$ chlorhexidine & -4.40 & 13.18 & 0.94 & -37.08 & 28.28 \\
\hline & Magnetized water & -5.20 & 13.18 & 0.9 & -37.88 & 27.48 \\
\hline \multirow[t]{2}{*}{ Magnetized water } & Magnetized $0.2 \%$ chlorhexidine & 0.80 & 13.18 & 0.99 & -31.88 & 33.48 \\
\hline & $0.2 \%$ chlorhexidine & 5.20 & 13.18 & 0.91 & -27.48 & 37.88 \\
\hline Magnetized $0.2 \%$ & Magnetized water & -0.80 & 13.18 & 0.99 & -33.48 & 31.88 \\
\hline chlorhexidine & $0.2 \%$ chlorhexidine & 4.40 & 13.18 & 0.94 & -28.28 & 37.08 \\
\hline \multicolumn{7}{|l|}{ Day 3} \\
\hline \multirow[t]{2}{*}{$0.2 \%$ chlorhexidine } & Magnetized $0.2 \%$ chlorhexidine & 3.20 & 9.09 & 0.93 & -19.36 & 25.76 \\
\hline & Magnetized water & -3.80 & 9.09 & 0.90 & -26.36 & 18.76 \\
\hline \multirow[t]{2}{*}{ Magnetized water } & Magnetized $0.2 \%$ chlorhexidine & 7.00 & 9.09 & 0.72 & -15.56 & 29.56 \\
\hline & $0.2 \%$ chlorhexidine & 3.80 & 9.09 & 0.909 & -18.76 & 26.36 \\
\hline Magnetized $0.2 \%$ & $0.2 \%$ chlorhexidine & -3.20 & 9.09 & 0.934 & -25.76 & 19.36 \\
\hline chlorhexidine & Magnetized water & -7.00 & 9.09 & 0.725 & -29.56 & 15.56 \\
\hline \multicolumn{7}{|l|}{ Day 7} \\
\hline \multirow[t]{2}{*}{$0.2 \%$ chlorhexidine } & Magnetized $0.2 \%$ chlorhexidine & 31.80 & 10.88 & $0.018^{*}$ & 4.81 & 58.78 \\
\hline & Magnetized water & -4.20 & 10.88 & 0.921 & -31.18 & 22.78 \\
\hline \multirow[t]{2}{*}{ Magnetized water } & Magnetized $0.2 \%$ chlorhexidine & 36.00 & 10.88 & $0.007^{*}$ & 9.01 & 62.98 \\
\hline & $0.2 \%$ chlorhexidine & 4.20 & 10.88 & 0.921 & -22.78 & 31.18 \\
\hline Magnetized $0.2 \%$ & $0.2 \%$ chlorhexidine & -31.80 & 10.88 & $0.018^{*}$ & -58.78 & -4.81 \\
\hline chlorhexidine & Magnetized water & -36.00 & 10.88 & $0.007^{*}$ & -62.98 & -9.01 \\
\hline
\end{tabular}

Test applied: Tukey's test; ${ }^{*} p \leq 0.05$ (Significant)

\section{Discussion}

Dental caries is a curable multi factorial disease, despite of credible scientific development, it continues to be a major public health problem in developing countries, changing dietary patterns and life-styles are strikingly increasing caries incidence.[11] According to World Health Organization, dental caries stands third amongst the most chronic non communicable diseases with 60 to $90 \%$ of prevalence among school going children.[12] 
Children belonging to age group 11-12 years are unique as they move on to higher grades in school, it represents the end point of caries history of deciduous teeth, and this age is the global standard for comparing the caries burden worldwide.[12-14] Unstimulated saliva was collected using spitting method as spitting seems to be most replicable and easy method to do so and unstimulated saliva represents the physiological basal flow of saliva on contrary to the stimulated saliva.[15,16] Saliva was collected in the morning between 10:00-12:00 am as this hour matched the naturally built in circadian rhythm of salivary flow.[17] All the children were asked to abstain from eating and drinking at least 90 minutes before collection. Sterile lab conditions and strict anaerobic/aerobic protocols was maintained to produces reliable results pertaining to the counting of the bacterial load. All the children were asked to rinse their mouth using the $10 \mathrm{ml}$ of the prepared mouth rinse once a day for 30 seconds and expectorate. All instruction given were in terms with the reference manual of useful medications for oral conditions as is the dose given for chlorhexidine gluconate above 8 years and was a maximum time up to which children could rinse without any discomfort.[18]

After the usage of the mouth washes, there was a reduction in count of streptococcus mutants after 3 days and significant reduction after 7 days. Chlorhexidine $(0.2 \%)$ mouth rinse was a gold standard, bacteriostatic at low concentration, bactericidal at high concentration and was also a potent antifungal agent, with pH. 4.24 and conductivity 10.72. According to the previous literature there was nearly $50 \%$ reduction in the number of streptococcus mutants after rinsing with $10 \mathrm{ml}$ of chlorhexidine once daily for a week.[2] Accredited mechanism stated by Lone et al(2016)[17] for plaque inhibition by chlorhexidine was blocking of acidic groups present on the salivary glycoproteins, reduces the protein adsorption by binding to the surface of the salivary bacteria polysaccharide coats, leads to interference of bacterial adsorption mechanism to the tooth surface. According to Leikin and Paloucek (2008), at physiologic $\mathrm{pH}$, chlorhexidine salts dissociate and the positively charged chlorhexidine cation.[19] Cations disrupts negatively charged bacterial cell wall by binding with it causing membrane disruption results into cell death. Although, chlorhexidine mouth washes are considered to be the gold standard for the stopping the plaque accumulation,[20] they should be used judiciously as they have potential toxic effect on the gingival fibroblast[21] and it also produced slight taste alteration.[22] In our study subjects baseline mean value of saliva sample was $233.8 \pm 33.34$, which reduced after rinsing with 72 hours magnetized water to $206.4 \pm 22.76$ and $185.8 \pm 22.54$ after 3 days and 7 days respectively, which was in accordance to previous study done by Gupta and Bhat (2011)[8] who used 72 hours magnetized water as mouthwash for a period of 1 week. According to Vangra (2008) magnetic field dissociate some molecules of water into hydrogen ion $(\mathrm{H}+)$ and hydroxyl ion (OH-).[23] Some of the hydroxyl ions will then combines with minerals such as calcium present in water and become calcium bicarbonate (salts) which has alkaline property, which can prevent the development of anaerobic microbes viz; $S$. mutans and also stops their growth.

According to Gupta and Bhat (2011)[8] the free oxygen ions of magnetized water can stop free radical cycle thus exhibiting antioxidant property which is directly related to the negative $\mathrm{pH}$ of the magnetized water (7.6 to 8.5 ). The water molecules enlarge in size, which increases the ability to dispense and penetrate other substances. So, the free radicals easily penetrate microorganism and aid in annihilation of bacteria. Magneto hydrodynamics prevents naturally occurring mineral deposition in fluids. [24] According to Hibben (1973), the bacterial inhibition produced by magnetized water occurs due to the interruption in the electrovalent bonding of cat ions, which prevents microorganism binding to a host surface.[25] Thus leading to inhibition of the plaque and calculus accumulation on the teeth surface.

In a study done by Kohno et al (2000), who evaluated the effect of ferrite magnet on the three strains of bacteria viz; S. mutans, S. aureus and E. coli, have found that increased strength of the magnetic field brought decrease in the growth rate of the bacteria.[10] The present study also revealed the same result.

There is scarcity of the literature in dentistry pertaining to the investigations related to antimicrobial efficacy of 72 hours magnetized $0.2 \%$ chlorhexidine mouthwash and there is no such literature found where this oral preparation has been used as a mouthwash either in-vitro or in-vivo. Thus, the present study is one of its kind, which evaluated the efficacy of this magnetized solutions as potential agent to be used as mouthwash in children. The 72 hours magnetized $0.2 \%$ chlorhexidine mouth wash having $\mathrm{pH} .5 .63$ and conductivity 12.40 as compared to $0.2 \%$ chlorhexidine which has $\mathrm{pH} .4 .24$ and conductivity 10.72 . In present study baseline mean value of saliva sample for 72 hours 
magnetized $0.2 \%$ chlorhexidine mouth wash was $233.0 \pm 27.17$, which reduced to $199.4 \pm 13.56$ and $149.8 \pm 27.47$ after 3 days and 7 days respectively and the reduction was found to be significant statistically on $7^{\text {th }}$ day from baseline.

Under the influence of magnetic field, the dissociation of chlorhexidine salts accelerates and leads to rapid release of cat ions causing disruption of bacteria at faster pace. Since magnetized chlorhexidine is more alkaline and $S$. mutans is anaerobic bacteria so, it reduces $S$. mutans adsorption to the tooth surface by blocking bacteria favoring acidic groups and its alkaline $\mathrm{PH}$ decreases the count of bacteria by stopping their growth. Hence, 72 hours magnetized $0.2 \%$ chlorhexidine mouth wash showed significant reduction in CFU/ml counts of S. mutans after 7 days. Thus, magnetically activated solutions have been shown to have broad-spectrum antibacterial activity. Magnetized solutions hinder the normal colony formation of the bacteria by disturbing the bonding of the cat ion molecules, thus inhibiting the deposition formation on the tooth surface. Magnetized solutions have the potential to be widely adopted within the healthcare environment due to its ease of production, environmental compatibility, rapid disinfection time, low-cost raw material requirement, limited toxicity, little operator skills and anti-inflammatory action.

\section{Conclusion}

Magnetically activated $0.2 \%$ chlorhexidine is a potent mouth wash for the reducing the burden of caries causing bacterial count. Magnetized water is as effective as a normal mouthwash against $S$. mutans and it more efficient than saliva alone for hindering the plaque accumulation. It can be safely suggested that it can be used as an alternative to commercially available mouthwashes as it is environmental friendly and economical.

\section{Financial Disclosure: Nil.}

Conflict of Interest: None declared.

\section{References}

1. Tanner ACR, Kressirer CA, Rothmiller S, Johansson I, Chalmers NI. The caries microbiome: Implications for reversing dysbiosis. Adv Dent Res 2018;29(1):78-85 doi:10.1177/0022034517736496

2. Sadat Sajadi F, Moradi M, Pardakhty A, Yazdizadeh R, Madani F. Effect of fluoride, chlorhexidine and fluoride-chlorhexidine mouthwashes on salivary Streptococcus mutans count and the prevalence of oral side effects. J Dent Res Dent Clin Dent Prospects 2015;9(1):49-52 doi:10.15171/joddd.2015.010

3. Lakade LS, Shah P, Shirol D. Comparison of antimicrobial efficacy of chlorhexidine and combination mouth rinse in reducing the
Mutans streptococcus count in plaque. J Indian Soc Pedod Prev Dent 2014;32(2):91-96 doi:10.4103/0970-4388.130780

4. Bansal A, Marwah A, Nigam AG, Goenk P, Goel D. Effect of Achyranthes aspera, $0.2 \%$ aqueous chlorhexidine gluconate and Punica granatum oral rinse on the levels of salivary Streptococcus mutans in 8 to 12 years old children. J Contem Dent Pract 2015;16(11):903-909

5. Ebrahimi A, Hemati M, Habibian Dehkordi S, et al. Chlorhexidine digluconate effects on planktonic growth and biofilm formation in some field isolates of animal bacterial pathogens. Jundishapur J Nat Pharm Prod 2014;9(2):e14298 doi:10.17795/jjnpp-14298

6. Umar D, Dilshad B, Farhan M, Ali A, Baroudi K. The effect of pomegranate mouthrinse on Streptococcus mutans count and salivary $\mathrm{pH}$ : An in vivo study. J Adv Pharm Technol Res 2016;7(1):13-16 doi:10.4103/2231-4040.173266

7. Forsythe J. The miracle of biomagnetic water. Ezine articles 2007. Available at: https://EzineArticles.com/expert/Janet_ Forsythe/114566. Accessed February 4, 2021

8. Gupta N, Bhat M. Comparative evaluation of 0.2 percent chlorhexidine and magnetized water as a mouth rinse on Streptococcus mutans in children. Int J Clin Pediatr Dent 2011;4(3):190-194 doi:10.5005/jp-journals-10005-1108

9. Hari AR, Water - a miracle therapy magnetized water, 1st ed. New Delhi: Pustak Mahal; 2008

10. Kohno M, Yamazaki M, Kimura I, Wada M. Effect of static magnetic fields on bacteria: Streptococcus mutans, Staphylococcus aureus, and Escherichia coli. Pathophysiology 2000;7(2):143-148 doi:10.1016/s0928-4680(00)00042-0

11. Basha S, Swamy HS. Dental caries experience, tooth surface distribution and associated factors in 6- and 13- yearold school children from Davangere, India. J Clin Exp Dent 2012;4(4):e210-e216 doi:10.4317/jced.50779

12. Petersen, Poul Erik, Baez, Ramon J, World Health Organization, Oral health surveys: basic methods, 5th ed. Geneva: World Health Organization; 2013

13. Gupta $H$, Hardiya S. Evaluation of efficacy of $2 \%$ povidone iodine and chlorhexidine in reducing salivary Streptococcus mutans. J Evolution Med Dent Sci 2014;3(72):15214-15220

14. Vissink A, Wolff A, Veerman E, Saliva collectors. In: David T, Wong, Ed. Salivary diagnostics. New Delhi: Wiley-Blackwell; $2008: 37-56$

15. Nayak SS, Ankola AV, Metgud SC, Bolmal U. Effectiveness of mouthrinse formulated from ethanol extract of Terminalia chebula fruit on salivary Streptococcus mutans among 12 to 15 year old school children of Belgaum city: a randomized field trial. J Indian Soc Pedod Prev Dent 2012;30(3):231-236 doi:10.4103/0970-4388.105016

16. Navazesh M, Kumar SK. Measuring salivary flow: challenges and opportunities. J Am Dent Assoc 2008;139 Suppl:35S-40S doi:10.14219/jada.archive.2008.0353

17. Lone N, Sidiq M, Khan M, Shah AF, Yousuf A. Short term effects of magnetised water and chlorhexidine on plaque accumulation and gingival inflammation - a randomized clinical study. Ann Int Med Dent Res 2016;2(3):91-94 
18. American Academy of Pediatric Dentistry. Useful medications for oral conditions. The Reference Manual of Pediatric Dentistry. AAPD 2020-2021:597

19. Leikin JB, Paloucek FP. "Chlorhexidine Gluconate", Poisoning and Toxicology Handbook, 4th ed. New York: Informa; 2008

20. Ulkur F, Arun T, Ozdemir F. The effects of three different mouth rinses in a 4-day supragingival plaque regrowth study. Eur J Dent 2013;7(3):352-358

21. Verma UP, Gupta A, Yadav RK, Tiwari R, Sharma R, Balapure AK. Cytotoxicity of chlorhexidine and neem extract on cultured human gingival fibroblasts through fluorescence-activated cell sorting analysis: An in-vitro study. Eur J Den 2018;12(3):344-349
22. Ripari F, Cera A, Freda M, Zumbo G, Zara F, Vozza I. Tea Tree Oil versus Chlorhexidine Mouthwash in Treatment of Gingivitis: A Pilot Randomized, Double Blinded Clinical Trial. Eur J Dent 2020;14(1):55-62

23. Vangra W. Property and Benefit of Magnetized Water. Ezine articles 2008. Available at: https://EzineArticles.com/expert/ Wevangti_Vangra/157538. Accessed February 4, 2021

24. K Goyal A, S Rathore A, Garg M, Mathur R, Sharma M, Khairwa A. Effect of magnetized water mouthrinse on Streptococcus mutans in plaque and saliva in children: An in vivo study. Int J Clin Pediatr Dent 2017;10(4):335-339 doi:10.5005/jp-journals-10005-1461

25. Hibben SO. Magnetic treatment of water. National Technical Information Services no. AD-757887 1973.:1622-1624 